\title{
A Transition State Theory Perspective on the Relation of Reversible Metal Hydride First-Order Kinetics to Equilibrium Thermodynamics
}

\author{
Roland H. Pawelke
}

\begin{abstract}
In the event of hydrogen desorption from reversible metal hydrides, equilibrium thermodynamics exert a rate-limiting effect: if system pressure reaches equilibrium pressure, the reaction rate becomes zero. This is usually dealt with by an empiric term of either polynomial or logarithmic nature to first-order kinetics. This paper approaches the matter from a transition state theory perspective, combining the classic Eyring-Polanyi equation with insights on reversible metal hydride chemical overpotential for scrutinizing the relation of Arrhenius first-order kinetics to van't Hoff equilibrium pressure. The outcome, tested for the example of $4 \mathrm{~mol} \%$ Ti-doped $\mathrm{NaAlH}_{4}$, suggests theoretical coherency and provides a method for identifying the factor by which an experiment deviates from ideal first-order kinetics. Adopting Arrhenius-Eyring-Polanyi first-order kinetics as baseline for modelling kinetic behaviour of metal hydride sorption reactions not only covers a blind spot in the Arrhenius approach but creates a standard for result comparability.
\end{abstract}

\section{Introduction}

The decomposition kinetics of reversible metal hydrides show by principle saturation behaviour with the reaction rate decreasing to zero if system pressure reaches metal hydride equilibrium pressure at the given temperature. This effect may be accounted for by amending first-order kinetics with a term of either polynomial or logarithmic nature, ${ }^{1-3}$ for the latter $\ln \left(p_{\text {eq }} / p\right)$ is an example of certain prominence. It may be the simplest but not necessarily the best solution and by principle, there are innumerable possibilities for an In-term equalling 1 at parity of applied and equilibrium pressure such as $\ln \left(2-p_{\text {eq }} / p\right)$ or $\ln \left(p_{\text {eq }} / p\right) \cdot \ln \left(99-98 p_{\text {eq }} / p\right)$. For thermodynamics and kinetics are commonly seen as functionally separate entities (the former tell about reaction viability, the latter about the speed of viable reactions), there is by principle no inner criterion for assessing a solution's quality.

Following some Ockham Razor line of argument, ${ }^{4-6}$ it may be stated that if in doubt, the simplest sufficient option is the best, which indeed favours $\ln \left(p_{\mathrm{eq}} / p\right){ }^{1}$ This is to a certain extent supported by notions about the ideal gas chemical potential and equilibrium thermodynamics yet LozANO et al state that for $\left[\mathrm{AlH}_{6}\right]$-decomposition in the $\mathrm{Ti}^{-} \mathrm{NaAlH}_{4}$ system this term is not the best for fitting their data and present a polynomial solution instead. ${ }^{3}$ It may be argued further that a first-order kinetics approach is not suited for this kind of problem altogether which is insofar not true as it can be used rather well if system pressure is substantially lower than equilibrium pressure. ${ }^{3,7}$

Hence it looms that an idea for untangling these intertwined strands without hiding any is due and while the issue may be satisfactorily resolved in an experiment-specific, external, empiric way, even in that case a diffuse feel of incompleteness remains: In essence, this is about first-order kinetics, a very fundamental matter and not about exploring some far-out unknown unknowns in R\&D or in Ockham's case, disputing about Christian metaphysics and miracles. If thought about closely, it shows that an empiric solution for the issue is rather easy at hand but much less so is an answer of wider scope. Hence it is worthwhile to re-think the issue by the following question: How to obtain an inner principle-based criterion for separating the thermodynamic equilibrium effect from first-order kinetics? It appears that this question has not been duly asked in relevant literature to date and for win, lose or draw in any case the outcome will contribute towards a better understanding of the art. 


\section{Methodical Approach}

Since this problem sits at the border between kinetics and thermodynamics, the classic EyringPolanyi equation is a suitable starting point. Originating from transition state theory (TST), ${ }^{8}$ it is founded on the axiomatic postulates that $a$ ) absolute reaction rates can be calculated from $b$ ) a fast anterior equilibrium of the reactants with an activated complex, in which c) the reaction products are already virtually present to be $d$ ) released in a rate-determining decomposition step.

It is by principle possible to think the problem from either reaction end of the reversible system but desorption has the advantage of the clearer boundaries: hydrogenation may occur at any pressure above the equilibrium pressure (facultative formation overpotential left aside), also affecting the reaction rate itself, but desorption pressure is clearly confined between zero and the equilibrium pressure. Actually, the reference to metal hydrides is a bit too specific: more befitting the generality of thermodynamics is the term reversible chemical hydrogen sorbent but metal hydride terminology is kept for reasons of prominence, brevity and example case. Equation 1 is a generic first-order reaction model for the decomposition of a reversible metal hydride $\mathrm{MH}_{\mathrm{x}}: \mathrm{M}$ may represent the metallic or a sub-hydride stage of the system.

$\mathrm{MH}_{\mathrm{x}} \rightleftharpoons\left[\mathrm{MH}_{2}\right]^{\ddagger} \rightleftharpoons \mathrm{M}+\mathrm{x} / 2 \mathrm{H}_{2}$

Equation 1 displays the reversible release of hydrogen according to the fundamentals of the EyringPolanyi equation: the metal hydride $\mathrm{MH}_{\mathrm{x}}$ is in fast equilibrium with the activated complex $\left[\mathrm{MH}_{2}\right]^{\ddagger}$ which collapses in a rate-determining decomposition step into the reaction products. Based on this image, two initial notions converge towards the kinetic model: First, at the macroscopic level the reaction follows first-order kinetics; the reaction rate is the product of a temperature-dependent constant $k$ with the metal hydride concentration $\left[\mathrm{MH}_{\mathrm{x}}\right]$. Second, at the molecular level the reaction rate is given by the collapse of the activated complex $\left[\mathrm{MH}_{2}\right]^{\ddagger}$ due to a vibration of the frequency $v$ along the reaction coordinate, leading to product formation. This is shown in equations $2 a$ and $2 b$.

Reaction rate $=k\left[\mathrm{MH}_{\mathrm{x}}\right]$

Reaction rate $=v\left[\mathrm{MH}_{2}\right]^{\ddagger}$

In order to account for the influence of equilibrium pressure on the hydrogen release rate, a factor $y$ is introduced to the right side of equation $2 \mathrm{~b}$. It is equation $2 \mathrm{~b}$ because in TST terminology, this thermodynamic pressure-dependent equilibrium term $y$ may be interpreted as a transmission factor accounting for the effective proportion of vibration events leading to product formation. Equations $3 a$ and $3 b$ show that accordingly.

Reaction rate $=k\left[\mathrm{MH}_{\mathrm{x}}\right]$

Reaction rate $=y v\left[\mathrm{MH}_{2}\right]^{\ddagger}$

Factor $y$ is for the moment not specified beyond the requirement that for system pressure $p$ approaching metal hydride equilibrium pressure $p_{e q}$ the hydrogen release reaction rate must approach zero. Equation 4a shows the law of mass action for the equilibrium between the activated complex and the starting hydride phase. Equation $4 \mathrm{~b}$ is the relation for the shifting of equilibrium composition in relation to the molar standard free enthalpy $\Delta G_{\mathrm{m}}{ }^{\circ \neq}$. Equation $4 \mathrm{c}$ expresses the concentration of the activated complex $\left[\mathrm{MH}_{2}\right]^{\ddagger}$ by means of equations $4 \mathrm{a}$ and $4 \mathrm{~b}$. 
$K^{\ddagger}=\frac{\left[\mathrm{MH}_{2}\right]^{\ddagger}}{\left[\mathrm{MH}_{\mathrm{x}}\right]}$

$\Delta G_{\mathrm{m}}{ }^{\circ \ddagger}=-\mathrm{R} T \ln \left(K^{\ddagger}\right)$

$\left[\mathrm{MH}_{2}\right]^{\ddagger}=\left[\mathrm{MH}_{\mathrm{x}}\right] \mathrm{e}^{-\Delta G_{\mathrm{m}}^{\circ} \neq / R T}$

Setting equations $3 a$ and $3 b$ equal yields equation $5 a$ and insertion of equation $4 c$ allows eliminating any concentration-related quantity as shown in equation $5 b$.

$k\left[\mathrm{MH}_{\mathrm{x}}\right]=y v\left[\mathrm{MH}_{2}\right]^{\ddagger}$

$k\left[\mathrm{MH}_{\mathrm{x}}\right]=y v\left[\mathrm{MH}_{\mathrm{x}}\right] \mathrm{e}^{-\Delta G_{\mathrm{m}} \neq / R T} \quad \Rightarrow \quad k=y v \mathrm{e}^{-\Delta G_{\mathrm{m}} \neq / R T}$

The next quantity to specify is the vibration frequency $v$, by TST it is given by the universal frequency factor $\mathrm{k}_{\mathrm{B}} T / \mathrm{h}: \mathrm{k}_{\mathrm{B}}$ and $\mathrm{h}$ are the Boltzmann and Planck constants, respectively. This leads to the classic Eyring-Polanyi equation (with the exception of transmission factor $y$ ), visible in equation 6 .

$k=y \frac{\mathrm{k}_{\mathrm{B}} T}{\mathrm{~h}} \mathrm{e}^{-\Delta G_{\mathrm{m}}{ }^{\circ} / \mathrm{R} T}$

In equation 6 , the free enthalpy $\Delta G_{\mathrm{m}}{ }^{\circ \neq}$ is the quantity commonly sought for subsequently deducing conclusions about the transition state. However, it is different here because $\Delta G_{\mathrm{m}}{ }^{\circ}$ is for reversible metal hydrides equal to the overpotential caused by kinetic hindrance which can be calculated from Arrhenius and van't Hoff parameters. ${ }^{9}$ Consequently, $\Delta G_{\mathrm{m}}{ }^{\circ}$ is expressed in terms of an ideal gas chemical potential at overpotential pressure $p^{*}$ and temperature $T^{*}$ as in equation $7, \Delta G_{\mathrm{m}}{ }^{\circ}<0$ being a basic requirement to the formation of the activated complex.

$\Delta G_{\mathrm{m}}{ }^{\circ \neq}=-\mathrm{R} T^{*} \ln \left(\frac{p^{*}}{\mathrm{p}^{\circ}}\right)$

If equation 7 is inserted into equation 6 for temperature $T=T^{*}$, the expression simplifies much and a specific reaction rate $k^{*}$ is obtained as shown in equation 8 .

$k^{*}=y \frac{\mathrm{k}_{\mathrm{B}} T^{*}}{\mathrm{~h}} \mathrm{e}^{-\left(-\ln \left(p^{*} / p^{\circ}\right)\right)}=y \frac{\mathrm{k}_{\mathrm{B}} T^{*}}{\mathrm{~h}} \mathrm{e}^{\ln \left(p^{*} / \mathrm{p}^{\circ}\right)}=y \frac{\mathrm{k}_{\mathrm{B}} T^{*}}{\mathrm{~h}} \frac{p^{*}}{\mathrm{p}^{\circ}}$

Because his specific Eyring-Polanyi rate $k^{*}$ is located at the peak of the activation energy, it is in Arrhenius terms equivalent to the pre-exponential factor $k_{0}$ (equation 9a) and termed accordingly $k_{0, E P}$ as shown in equation $9 \mathrm{~b}$.

$k=k_{0} \mathrm{e}^{-E_{a} / R T}$

$k^{*}=y \frac{\mathrm{k}_{\mathrm{B}} T^{*}}{\mathrm{~h}} \frac{p^{*}}{\mathrm{p}^{\circ}}=k_{0, \mathrm{EP}}$

Equation $9 \mathrm{~b}$ shows the sought relation of inner principle for it shows by means of the shift in $k_{0}$ values the deviation of experimental data from theoretically ideal first-order kinetics. The difference may be interpreted as caused by equilibrium thermodynamics taking effect on kinetics. This provides a fixed point to factor $y$ which may be then modelled in polynomial or logarithmic manner for specific data fitting. Considering the variability of experimental setups, amending the Arrhenius approach by the Eyring-Polanyi theory covers a blind spot and recommends itself as standard to metal hydride kinetic models. 


\section{Results}

Equation $9 \mathrm{~b}$ is tested against the example of the $\left[\mathrm{AlH}_{4}\right]$-stage in the $4 \mathrm{~mol} \%$ Ti-doped $\mathrm{NaAlH}_{4}$ system. The quantities $T^{*}$ and $p^{*}$ relate to chemical overpotential and originate from other work, ${ }^{9}$ basing on the activation energy of $80 \mathrm{~kJ}\left(\mathrm{~mol} \mathrm{H}_{2}\right)^{-1}$ by SANDROCK et al and the van't Hoff parameters $\Delta H_{\mathrm{m}}{ }^{\circ}=+37$ $\mathrm{kJ}\left(\mathrm{mol} \mathrm{H}_{2}\right)^{-1} \Delta \mathrm{S}_{\mathrm{m}}{ }^{\circ}=+121 \mathrm{~J}\left(\mathrm{~mol} \mathrm{H}_{2}\right)^{-1} \mathrm{~K}^{-1}$ by Bogdanović et al, ${ }^{7,10}$ equation 10 shows accordingly.

$k_{0, E \mathrm{E}}=y \frac{\mathrm{k}_{\mathrm{B}} T^{*}}{\mathrm{~h}} \frac{p^{*}}{\mathrm{p}^{\circ}}=y \cdot 1.62 \cdot 10^{11} \mathrm{~h}^{-1}$

$p^{*}=78.7$ bar $\quad T^{*}=355 \mathrm{~K} \quad \mathrm{k}_{\mathrm{B}}=1.380649 \cdot 10^{-23} \mathrm{~J} \mathrm{~K}^{-1} \quad \mathrm{~h}=6.62607015 \cdot 10^{-34} \mathrm{~J} \mathrm{~s}=2.385385254 \cdot 10^{-30} \mathrm{~J} \mathrm{~h}$

\section{Discussion}

Since the result of equation 10 bases on the activation energy value of SANDROCK et al, it must be seen in context to the corresponding experimental $k_{0}$ value which is $k_{0}=1.81 \cdot 10^{11}[\% \mathrm{w} / \mathrm{w} \mathrm{H}] \mathrm{h}^{-1}$, the formal adjustment to the mass percent hydrogen desorbed is trivial. It is noteworthy that the experimental setup of SANDROCK et al aims at minimizing equilibrium pressure effect by desorbing into an evacuated Sievert apparatus of such dimensions that final pressure always remains below 1 bar so first-order Arrhenius kinetics apply. This experiment is complementary to the Eyring-Polanyi approach outlined and the difference in $k_{0}$ values is just $11.7 \%$ : this small difference (which further still includes the margin of experimental error) and the near-ideal linear Arrhenius plot(s) suggests the experimental assumptions with regard to first-order kinetics being reasonably justified. ${ }^{1,11}$ Interestingly and contrary to the usual, this suggests using the comparatively objective EyringPolanyi $k_{0, \text { EP }}$ and not an experimental Arrhenius $k_{0}$ value as base for first-order kinetic model building. In contrast, the Arrhenius activation energy $E_{\mathrm{a}}$ may be used as obtained by e.g. the method of SANDROCK et $a$ l because it originates from a linear slope respective a quotient of differences and therefore is comparatively prone to a distortion caused by equilibrium thermodynamics.

Literature provides a couple of illustrative works for discussing this finding further in relation to the nature of factor $y$ : a subsequent follow-up study by LUO AND GROSs, ${ }^{1}$ using virtually the experimental setup of SANDROCK et al but introducing $\ln \left(p_{\text {eq }} / p\right)$ as factor in the data processing, arrives at in part very different results. ${ }^{1,7}$ It is rather debatable whether this step is an improvement as the resulting Arrhenius plots are not as convincingly linear as those presented by SANDROCK et al. Furthermore, LOZANO et al state that $\ln \left(p_{\text {eq }} / p\right)$ is not the best term for first-order fitting their data: ${ }^{3}$ albeit that is with regard to the $\left[\mathrm{AlH}_{6}\right]$-stage, it nonetheless suffices to raise doubts about its general applicability.

Said study of LOZANO et al conveys two further noteworthy points: first, it shows on the basis of the Johnson-Mehl-Avrami equation that first-order kinetics is only a valid assumption if system pressure is substantially below equilibrium pressure and give $1-p / p_{\text {eq }}<0.7$ as threshold. Yet the respective plot suggests that the threshold should be rather at 0.6 than 0.7 for clear linearity which insofar supports a logarithmic base relation as any e- respective In-function may be subdivided into two virtually linear parts by the golden section, thus 0.618 and 0.382 . Second, the term $\ln \left(p_{\text {eq }} / p\right)$ is found not to be the best solution for first-order fitting of their $\left[\mathrm{AlH}_{6}\right]$ desorption data and the polynomial solution $\left(\left(p_{\text {eq }}-p\right) / p_{\text {eq }}\right)^{2}-0.46\left(\left(p_{\text {eq }}-p\right) / p_{\text {eq }}\right)$ is presented instead. This term may fit the respective data well but it is still an empiric solution and as such of dubious general applicability, eventually it yields just another set of experimental Arrhenius parameters. This highlights the limitation of the hitherto case-specific practice of measuring work quality in terms of fitting own experimental data at little to no real comparability beyond. Due to the empiric nature of the Arrhenius approach, the resulting parameters always merely reflect the prerequisites by which they had been obtained, the studies of LUO AND GROSS and SANDROCK et al show that in most instructive manner. ${ }^{1,7}$ 
This underscores the benefit from a scientific point of view of introducing a standard to kinetic models for reversible metal hydrides and Arrhenius-Eyring-Polanyi first-order kinetics recommend itself for that role. It is noteworthy that the TST conception outlined for desorption shows also in the absorption reaction as well. A preceding study of thermodynamic scope concerned with the volume expansion upon hydrogenation arrives (for the example of $\mathrm{K} / \mathrm{Ti}$-co-doped $\mathrm{NaAlH}_{4}$ ) at the finding that the molar volume of the gas phase must be at least equal to the average molar volume of the hydride phases active in the absorption process. ${ }^{11}$ Though not within intent and not realized at the time, this is a reflection of the TST postulate that the reaction product is virtually present in the activated complex prior its actual formation. The unexpected, independent confirmation of the findings from this study by one of thermodynamic scope and complementary variable (molar volume) on the same base system may be seen as a token for the fundamental correctness of both.

\section{Conclusions}

Combining insight about reversible metal hydride overpotential with the Eyring-Polanyi equation not only elucidates the relation of desorption first-order kinetics to equilibrium thermodynamics but rewards with a structured approach towards first-order kinetic models for reversible metal hydrides:

\$1 Arrhenius and van't Hoff parameters are fundamental; with regard to the former, a dedicated first-order experimental setup is indispensable for which the work of SANDROCK et al may serve as a reference; ${ }^{12}$ on that basis the reversible metal hydride overpotential may be calculated. ${ }^{9}$

§2 The overpotential is instrumental for determining the ideal first-order Eyring-Polanyi $k_{0, \mathrm{EP}}$ parameter (this work) which is shifted by a factor $y$ from the experimental Arrhenius $k_{0}$ value of $\S 1$. The shift between theoretical first-order $k_{0, \mathrm{EP}}$ and experimental $k_{0}$ Arrhenius may be interpreted as the residual impact of equilibrium thermodynamics in the experimental setup of $\S 1$. Consequently, it is this $k_{0, \mathrm{EP}}$ and not an experimental Arrhenius $k_{0}$ value which in combination with the under $\S 1$ determined $E_{\mathrm{a}}$ value should make the base for modelling first-order kinetics. $E_{\mathrm{a}}$ can be used for it is derived from a quotient of differences and as such less prone to thermodynamic distortions.

§3 Such an Arrhenius-Eyring-Polanyi (AEP) first-order approach recommends itself as a general baseline for metal hydride kinetic models.

$\S 4 \quad$ Impact of equilibrium thermodynamics on AEP first-order kinetics may be modelled by means of factor $y$ (equation $9 \mathrm{~b}$ ). The resulting term can be either of polynomial or logarithmic nature; the latter is suggested on the basis of work by LozANO et al, in contrast to their own interpretation. ${ }^{3}$ The term $y=x \ln \left(z-\left((z-1) p_{\text {eq }} / p\right)\right.$ is suggested as a general template of which the simple term $\ln \left(p_{\text {eq }} / p\right)$ is the specific case for $x=1$ and $z=0$.

\$5 The TST conception outlined in this paper for desorption shows also in the absorption process as it has been found for $\mathrm{K} / \mathrm{Ti}$-co-doped $\mathrm{NaAlH}_{4}$ that the molar volume of the gas phase must be at least equal to the average of the hydride-phases active as a prerequisite to the absorption process, ${ }^{11}$ reflecting the postulate that the products are virtually present in the activated complex.

\section{Acknowledgements}

This paper spun-off from the author's primary line of work done at FOTEC Forschungs- und Technologietransfer GmbH under European Space Agency grant 4000105330/12/NL/CLP and European Defence Agency contract A-1341-RT-GP which is gratefully acknowledged. 


\section{Conflicts of Interest}

There are no conflicts of interest to declare.

\section{References}

1 W. Luo and K. J. Gross, J. Alloys Compd., 2004, 385, 224-231.

2 F. Yang, Y. Zhang, F. Ciucci, Z. Wu, S. Wang, Y. Wang and Z. Zhang, J. Alloys Compd., 2018, 741, 610-621.

3 G. A. Lozano, C. N. Ranong, J. M. Bellosta von Colbe, R. Bormann, G. Fieg, J. Hapke and M. Dornheim, Int. J. Hydrog. Energy, 2010, 35, 7539-7546.

4 W. M. Thorburn, Mind, 1915, XXIV, 287-288.

5 W. M. Thorburn, Mind, 1918, XXVII, 345-353.

6 J. Schaffer, Australas. J. Philos., 2015, 93, 644-664.

7 G. Sandrock, K. Gross and G. Thomas, J. Alloys Compd., 2002, 339, 299-308.

8 P. Ptáček, F. Šoukal and T. Opravil, in Introducing the Effective Mass of Activated Complex and the Discussion on the Wave Function of this Instanton, eds. P. Ptáček, T. Opravil and F. Šoukal, InTech, 2018.

9 R. H. Pawelke, On the Common Ground of Thermodynamics and Kinetics: How to Pin Down Overpotential to Reversible Metal Hydride Formation and the Complete Ideal Gas Theory of Reversible Chemical Hydrogen Storage, 2020.

10 B. Bogdanović, R. A. Brand, A. Marjanović, M. Schwickardi and J. Tölle, J. Alloys Compd., 2000, 302, 36-58.

11 R. H. Pawelke, How to Assess the Relative Volume Change of Reversible Metal Hydrides Easily, Speedily and Concisely (Enough) with a Surprising Relevance for Clarifying Thermodynamic Tailoring Effects, 2019.

12 G. Sandrock, K. Gross and G. Thomas, J. Alloys Compd., 2002, 339, 299-308. 